\title{
Norm manipulation as a condition of friendship
}

\section{Mark Phelan ${ }^{\mathrm{a} *}$}

${ }^{a}$ Department of Philosophy, Lawrence University, 711 East Boldt Way, Appleton, WI 54911, USA Email:mark.phelan@lawrence.edu 


\section{Norm manipulation as a condition of friendship}

Cathy Mason (2020) argues — against my position in Phelan (2019) — that significant norm-manipulation is unnecessary for friendship. Instead, she holds that norm manipulation is a, perhaps omnipresent, causal result of the very feature I deny as necessary to friendship: mutual caring or love. Mason's counter-examples allow for further explication of the normmanipulation view of friendship. However, they do not constitute a compelling challenge to that view, because they do not seem to involve collaborative norm manipulation at all. Instead, they are better described as cases in which people come to be subject to established cultural norms they were not previously subject to, because they voluntarily come to fall under a distinctive relationship relative to one another.

Keywords: Friendship, norms, norm manipulation 
Mason (2020) argues, against my position in Phelan (2019), that significant norm-manipulation is unnecessary for friendship. Instead, she holds that norm manipulation is a, perhaps omnipresent, causal result of the very feature I deny as necessary to friendship: mutual caring or love. She writes,

...even if norm-manipulation is a feature of all friendships, it may be that this is a merely incidental rather than basic feature of friendship, and one that is a consequence of the very kind of feature that Phelan initially rejects (3).

Mason proposes that I face a dilemma over whether the norms that get manipulated in the formation of friendship are "norms that apply to...individuals on the basis of some specific social position that they occupy in relation to one another" or "norms that apply to any set of strangers one encounters within a given cultural context" (3). She argues that my view is too demanding because 'social position' norms need not be manipulated in order for people to form friendships. And she argues that my view is too permissive because 'stranger norms' are sometimes collaboratively manipulated for reasons that are inappropriate to the formation of friendship.

I doubt there is a useful distinction to be drawn between social position norms and stranger norms. On my view, norms are expectations of how people do and are expected to behave that are self-enforcing. Such expectations are clearly contextually embedded - that is, they are dependent on background conditions for their specific structure. Often the background conditions on the basis of which a given norm has its structure will relate to the specific social position that two agents occupy in relation to one another. For example, it may be a social norm in some culture that women open the door for men. Our expectations, then, of how two strangers will behave relative to this norm as they approach a door are dependent on the social position that they occupy, relative to cultural gender stereotypes, relative to one another. Indeed, our expectations of how others will behave are, as a matter of descriptive fact, quite typically dependent on our impressions of their positionality - their socio-economic, gender, ethnic, age, occupational, transactional, and so on status. ${ }^{1}$ Because almost all non-manipulated normsincluding those operating among strangers — are social position norms, I will mostly ignore Mason's distinction going forward. But I will take up both of her challenges.

According to Mason, my view entails that "at least some norms that usually apply given their social positions must be manipulated in order for two individuals to become friends" (4). This is correct, with the caveat I have just been making, that almost all —if not all — norms apply given or relative to our social positions. Indeed, my argument for the claim that collaborative norm manipulation is a necessary feature of friendship depends on the idea that we don't typically think of purely conventional relationships as friendships. But Mason contends that this fails to hold for some friends that occupy special positions relative to one another, and that my view is therefore too narrow, since it leaves out some legitimate friendships. Using an example involving some work colleagues that I introduced, she writes:

It might be the case that such individuals sit closer to one another than they otherwise would, laugh at jokes that are generally socially frowned upon, or

\footnotetext{
${ }^{1}$ Notice, I am not claiming that all of our interactions are socially structured. It is possible to act in a way that departs from a normative framework. After all, an expectation is not an expectation if it cannot be broken.
} 
accept casual cursing... But there seems to be no reason to think that they would necessarily do this. They might well enjoy one another's company without their relationship involving any norms that go beyond those that customarily apply to work colleagues.

I agree that individuals in a thoroughly conventional relationship might enjoy one another's company. But this does not entail that they are friends, since we may enjoy the company of people who are not our friends. Mason needs to make the further case that a thoroughly conventional relationship might constitute a friendship. Nor is it enough to show a friendship between co-workers who have not violated any of the special cultural norms that govern the coworker relation. For although our relationships with our co-workers are in part normatively structured through the co-worker relation, any pair of co-workers are also subject to more general norms that would apply to those two individuals were they mere strangers. A female employee and a male employee embedded in a culture in which it is a general social norm that women open the door for men, may abide by this norm in addition to the special norms that apply to co-workers in their culture. It is consistent with my view, then, that a friendship might exist between two co-workers who have not violated any of the special norms that apply to them as co-workers, as it might at a company that prohibits workplace fraternization between employees. Such friends might have significantly manipulated the cultural norms that apply to them given their more general positionality relative to one another instead. However, it is ultimately a base level assumption of my view that two people whose expectations of one another do not differ from the expectations they have of mere co-workers, or even mere strangers, are not friends. But, then again, I don't think Mason is too far off from this position, since she concedes that "norm manipulation may be a feature of friendship, and perhaps even a ubiquitous one" (6). I turn to her second criticism.

I maintain that friendship is a relationship necessarily involving collaborative norm manipulation. Mason argues that if this were a sufficient condition (instead of just a necessary one), then my view would be too permissive, because "we can collaboratively manipulate norms for many different reasons, and many of these seem like the wrong kinds of reason to count as friends" (4). As an example of the sort of norm-manipulation that seems inappropriate to friendship, Mason provides a case of two individuals who "jointly decide that they would work well together as business partners" (5). She contends that this decision would necessitate manipulation of many norms. For example, business partners discuss their finances with one another and make demands of one another that would be wholly inappropriate were they mere strangers. Mason contends that, absent mutual liking, "we would not be at all tempted to think that the two business partners are friends" (5). According to Mason, I embrace my second, necessary 'mutual positive disposition condition' on friendship in order to rule out problem cases like these. As I will explain, this is not the reason I embrace that condition. I think that either the significant norm manipulation condition is sufficient to rule out Mason's problem cases or else we would be tempted to call such relationships friendships.

The relationships Mason describes do not seem to satisfy the significant norm manipulation condition. As discussed above, norms - other than the collaboratively-manipulated sort that are constitutive of friendship — are often structured by the relevant agents' social position. In fact, many cultural norms are 'in play' only when agents occupy specific relations to one another. For example, it is normative for tipping to occur during a restaurant meal in the 
United States, but this norm holds only between the diner and the server, not between the server and the bartender, say, or the diner and another diner. ${ }^{2}$ The business partners in Mason's example are like the server and the diner; they are best described as bound by existing cultural norms that apply to people who have entered into a specific social relationship. This is why Mason can justify their behaviour by appealing to their relationship: "After all, they are business partners" (5). It is true that the relationship between business partners is a sort of collaboration, just as my successfully ordering a meal at a restaurant is a sort of collaboration between me qua diner and my server. But in neither case do we collaboratively manipulate norms. In both we simply come to be subject to certain, established cultural norms we were not previously subject to because we voluntarily come to fall under a distinctive relationship relative to one another.

Now, it is true that two people - two business partners, perhaps - might come to collaboratively manipulate norms for merely strategic reasons, or, as Mason puts it, "based on a clear-eyed assessment of the compatibility of the other's expertise and skills, without any personal interest or feelings on either side" (5). For example, suppose that it is descriptively normative in some culture for business partners to jointly select the décor of their common work space. But suppose one of them is colour-blind and also self-conscious about her bad taste. She knows that clients will often come into the work space, so it is important that it be aesthetically pleasing. Based on a purely strategic, clear-eyed assessment of what is in her personal interest, this business partner may propose that the other solely handle decisions of work place décor. If the other partner accepts this shift in décor responsibility, the two will have jointly manipulated a small part of the normative structure that informs their relationship. They will have formed new expectations of one another in a collaborative way. This is a small change, but others might follow. One partner might pick up coffee one Monday morning. She may subsequently, tacitly propose to make it a regular thing that she brings coffee on Mondays by bringing it the next week again, to no objection. In collaborating to produce this norm, at least part of her motivation may well be the cold capitalist one that it seems to increase productivity. Nonetheless, this constitutes another stitch in a growing normative fabric. Perhaps, after time, one of the partners broaches discussions of her personal life with the other. She may do this, at least in part, because she suspects she has been underperforming as a business partner due to stresses at home. Her joint business endeavour is critically important to her and she worries that it will fail if she doesn't unburden herself to someone. If her partner listens openly the two may form a normative expectation to discuss personal matters, even though it is not culturally typical for business partners to do so. A significant number of such changes made in a similar way could eventually build a unique, central relationship in the lives of two people. It is my view that such a relationship could be well-described as a friendship, ${ }^{3}$ and at least partially in virtue of this unique normative structure. Thus, I disagree with Mason when she writes that being "governed by norms that have been collaboratively manipulated seems to tell us nothing at all about whether it is well-described as a friendship" (5). More generally, I disagree with Mason's assessment that some of the reasons for which we collaboratively manipulate norms are "the wrong kind of reasons to count as friends" (4). Our reasons for acting in general and for manipulating norms in particular are typically multifarious and complicated and often not known to us in their entirety.

\footnotetext{
2 This point is akin to Mason's that "there are many different social scripts for different contexts" (5), and Mason agrees that there are distinctive cultural norms in place for different specific relationships.

${ }^{3}$ Provided, of course, that the two people like one another, since I maintain that significant norm manipulation is but a necessary condition on friendship. More on this shortly.
} 
It seems ill-advised, then, to rule out certain motivations as incorrect at the outset. Moreover, there seem to be examples from film, literature and common life of friendships initially broached for self-interested, instrumental reasons. ${ }^{4}$

It is true that I embrace a second, necessary 'mutual positive disposition condition' on friendship — or, as Mason helpfully recasts it, a 'mutual liking condition'. I embrace it not to rule out problem cases, but simply because it seems incorrect to refer to people who do not like each other at all, in any respect, as friends. However, Mason argues that once I introduce this condition "it renders the norm-manipulation condition obsolete, because it plausibly explains norm manipulation" (6). Mason offers two considerations in favour of this idea. Mason's first consideration generalises from her claim that I must embrace a mutual liking condition in order to rule out instances of norm manipulation that are irrelevant to friendship, to the conclusion that the only instances of norm-manipulation that are relevant to friendship are those that are the product of mutual liking. But, as I have explained above, I need not invoke mutual liking to rule out such instances, nor are considerations of motivation sufficient to render instances of collaborative norm manipulation irrelevant to the formation of friendship.

Mason's second consideration rests on the idea that we ordinarily "take friendship to explain why people manipulate certain norms, rather than to be explained by it" (6). Mason usefully points out, for example, that people hug (and thereby manipulate norms of personal space) because they are friends, "whereas is would seem strange to say that two people are friends "because they hug"" (6). It is strange to say that two people are friends 'because they hug', but this is because hugging someone (in our culture) involves a relatively shallow manipulation along a single normative dimension, whereas my view is that friendships involve a significant degree of collaborative norm manipulation. It is far less strange to say that two people are friends because they are able to joke in socially prohibited ways with one another, because they are able to talk to one another about things one is supposed to keep private, because they expect to help each other out when there is no expectation that they should do so, and so on. Mason is surely correct that people collaboratively manipulate certain norms because they like one another, but we also sometimes like people because they have manipulated norms with us.

We do like our friends, of course. But mutual liking is clearly not sufficient for friendship, as people who mutually like one another are not always friends. In Phelan (2019), I argued that deep, mutual caring and significant intimacy are unnecessary for friendship. What then, in addition to mutual liking, makes for a friendship? Significant norm manipulation is a condition of friendship.

\footnotetext{
${ }^{4}$ Consider, for example, one of the most famous friendships in English literature, that between James Boswell and Samuel Johnson. More than three decades his junior, Boswell had desired to meet Johnson for reasons of personal edification for two years by the time they bumped into each other at Tom Davies's bookshop on May 16, 1763. In his London Journal, Boswell remarks, "about seven came in the great Mr. Samuel Johnson, whom I have so long wished to see" (220). A more contemporary, fictional example is, perhaps, the friendship between Joe Buck and "Ratso Rizzo" in Midnight Cowboy. Indeed, Aristotle (1999) suggests that true, character friendships might arise for instrumental reasons, since he contends that goodwill is the proper reaction to being benefitted, and that "goodwill is inactive friendship, and that when it lasts some time and they grow accustomed to each other, it becomes friendship" (1167a12).
} 
Acknowledgements: Ingrid Albrecht, Celia Barnes, Benjamin Chan, Kathy Mason, and Mark Warren provided feedback on earlier drafts that helped improve this paper.

\section{Works Cited}

Boswell, J., Morley, C., \& Pottle, F. A. (1950) Boswell's London journal, 1762-17633: Now first published from the original manuscript. (New York: McGraw-Hill.)

Mason, C. (2020) “On the Basis of Friendship” Inquiry.

Phelan, M. (2019) "Rethinking Friendship" Inquiry.

Aristotle. (1999) The Nicomachean Ethics. Translated by T. Irwin, 2nd ed. (Indianapolis, IN: Hacket Publishing.) 